based HEMT technology'. 2002 IEEE Int. Solid-State Circuits Conf. Tech. Dig., San Francisco, CA, USA, 2002, pp. 192-193

5 ISHI, K., MURATA, K., IDA, M., KURISHIMA, K., ENOKI, T., SHIBATA, T., and SANO, E.: 'Very-high speed selector IC using $\mathrm{InP} / \mathrm{InGaAs}$ heterjunction bipolar transistor', Electron. Lett., 2002, 38, (10), pp. 480-481

6 MURATA, K., OTSUI, T., SANO, E., KIMURA, S., and YAMANE, Y.: '70-Gbit/s multiplexer and $50-\mathrm{Gbit} / \mathrm{s}$ decision IC modules using InA!As/InGaAs/InP HEMTs', IEICE Trans. Electron., 2000, E83-C, (7), pp. 1166-1169

7 ENOKI, T, ITO, H., IKUTA, K., and ISHII, Y.: '0.1 $\mu \mathrm{m}$ InAlAs/InGaAs HEMT with an InP-recess-etch stopper grown by MOCVD'. Int Conf. on Indium phosphide and related materials, 1995, pp. 81-88 .

8 SANO, K., MURATA, K., and YAMANE, Y.: '50-Gbit/s demultiplexer IC module using InAlAs/InGaAs/InP HEMTs', IEICE Trans. Electron. 2000, E83-C, (11), pp. 1788-1790

\section{Fabrication of directly UV-written channel waveguides with simultaneously defined integral Bragg gratings}

G.D. Emmerson, S.P. Watts, C.B.E. Gawith, V. Albanis, M. Ibsen, R.B. Williams and P.G.R. Smith

A new technique for UV direct writing of Bragg gratings in plana silica is presented. In this method the Bragg gratings and the channels are defined simultaneously, conferring advantages in flexibility of design and grating performance. Photosensitive germanium-doped silica-on-silicon produced by flame hydrolysis deposition was used.

Introduction: Direct UV-writing is a fabrication process for planar waveguides based on the same permanent photoinduced refractive index change used in the fabrication of fibre Bragg gratings [1]. This UV induced refractive index change has recently been applied to the field of integrated optics, showing that an intense UV beam focused to form a writing spot and translated within the photosensitive core of a planar sample can form a low-loss channel waveguide [2]. Subsequent progress has demonstrated the suitability of direct writing to produce integrated optical components, including power splitters and directional couplers [3]. The integration of directly UV written waveguides and Bragg gratings within a planar geometry is of great significance, but to date directly written channels with Bragg gratings have bcen produced using a two-step process. In such cases, a primary exposure is often used to create a channel waveguide while a secondary exposure superimposes the grating structure. As the channel writing process often saturates the photosensitive response of the material a sequential process cannot be used to optimise both the grating strength and waveguide geometry in a single process. To this end we present a new method of directly writing a Bragg grating structure whilst simultaneously defining the channel waveguide, thus allowing the optimal use of the photosensitivity of the material.

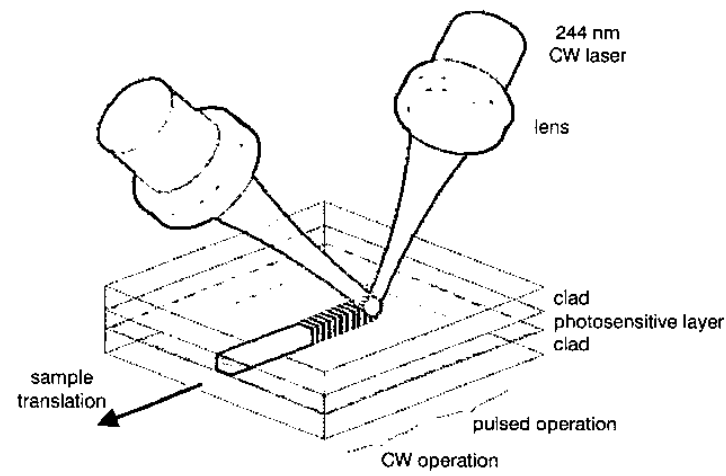

Fig. 1 Simultaneous direct UV writing of channel and subsequent grating sections using focused beam interference within core of photosensitive planar FHD sample

Experiment: For these initial experiments three-layer germaniumdoped silica-on-silicon samples developed at the Optoelectronics
Research Centre using flame hydrolysis deposition (FHD) were used as the writing media. Germanium doping of the middle layer introduces inherent photosensitivity to UV radiation [1], while all three layers were co-doped with phosphorus and boron facilitating independent control of refractive index and processing parameters.

Fig. 1 shows a schematic diagram of our approach. Direct UV writing into the buried germanosilicate layer was performed using a $\mathrm{CW}$ frequency doubled argon-ion laser operating at $244 \mathrm{~nm}$. A beam splitter was used to create two separate beam paths at an intersection angle of $27^{\circ}$ and each beam was individually focused to a spot size of $6 \mu \mathrm{m}$. The two focused beams are crossed within the photosensitive layer resulting in a writing spot with an inherent interference pattern. During writing this interference allows a channel waveguide containing a periodic refractive index modulation within the photosensitive layer to be written.

When the sample is translated under a constant writing spot the intraspot intensity pattern is averaged out, resulting in a channel waveguide. However, if the laser beam intensity is modulated with a period close to the time taken for the sample to move one grating period through the intra-spot interference, then a channel waveguide is induced that has a periodic index modulation, thus forming a Bragg grating. In our system the laser beam is pulsed using an acoustic-optic modulator triggered by an interferometer monitoring the position of the sample. The formation of Bragg gratings within the core can be directly controlled by changing the spatial superposition of the interference pattern, as is utilised in the fabrication of continuous fibre Bragg gratings [4]. This technique allows different grating periods to be defined without the need to change the beam intersection angle. The period of the Bragg grating is defined by the laser modulation as the sample is translated, and not solely by the period of the interference pattern. The range of detuning allowed depends inversely upon the number of planes in the writing spot and so a small spot is advantageous in writing widely detuned gratings. In this Letter we are presenting preliminary results on Bragg gratings of varying period, length and strength.

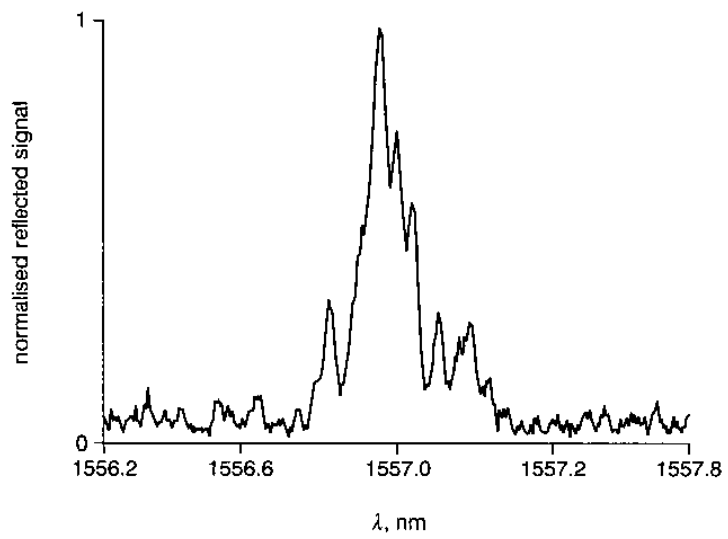

Fig. 2 Typical reflection spectra for simultaneously defined planar Bragg grating

Results: The gratings were analysed using an EDFA based ASE source, the output of which was transmitted and selectively orientated through a fibre polariser. Connected to the polariser was a $3 \mathrm{~dB}$ coupler to allow the back-reflected signal to be analysed using an OSA. Fabry-Perot resonances were partially suppressed through use of index matching fluid at the point of fibre launch.

An example of the back-reflected signal from a $14 \mathrm{~mm}$-long grating is shown in Fig. 2 and exhibits a reflectivity of approximately $28 \%$ with a FWHM of $0.132 \mathrm{~nm}$. As can be seen, the spectral response is not optimised and exhibits Fabry-Perot fringes due to stray reflections from the sample facets. Future devices are planned that will incorporate chirped and apodised grating structures with optimised UV induced index, thereby allowing higher reflectivity and tailored spectral response.

Fig. 3 shows the effect of the period of the grating written against the peak reflected wavclength. As expected from the Bragg condition the response is linear, allowing for $n_{\text {eff }}$ of the channel and grating to be measured to a high degree of accuracy. Precise control of the polarisa- 
tion state launched into the sample allowed the birefringence of the structure to be obtained, $n_{\text {eff }}$ for TE and TM polarisation states were 1.46597 and 1.46594, respectively, implying a of birefringence of $3 \times 10^{-5}$. Fig. 4 shows $n_{e f f}$ for a range of different waveguides written at different fluences by varying the translation speed of the sample.

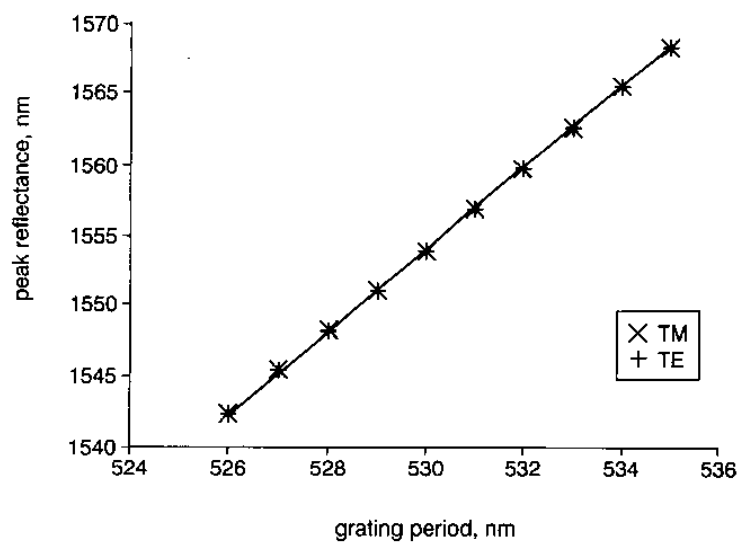

Fig. 3 Range of grating periods and associated measured peak reflection wavelengths, allowing determination of $n_{\text {eff }}$ for TE and TM polarisation

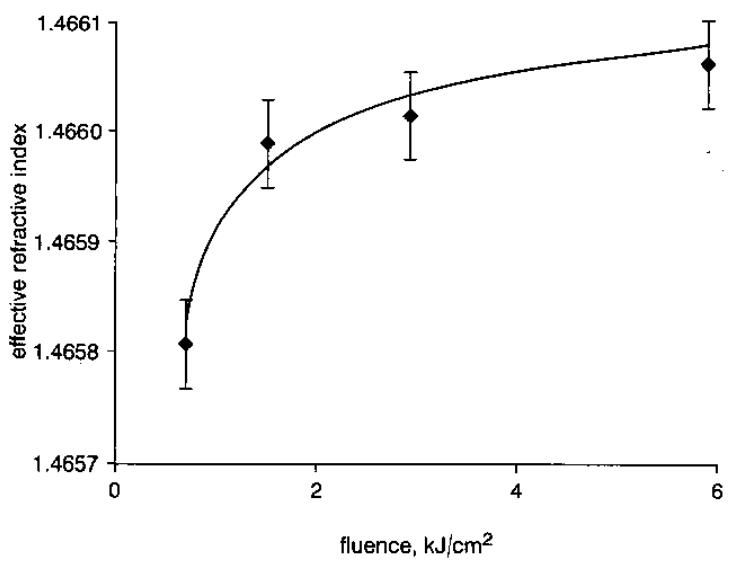

Fig. 4 Variation of $n_{\text {eff }}$ with writing fuence, varied by altering sample translation speed

Discussion: We have demonstrated the first simultaneously written channels with integral Bragg gratings in a photosensitive planar media. We have shown that it is possible to write Bragg gratings over a large range of wavelengths by pulsing of the writing spot intensity during translation entirely under software control. Based on these initial results, future FHD samples will be optimised for UV writing and photosensitivity will be enhanced through the investigation of deuterium loading, selective dopant concentrations and other associated processes. The future development and optimisation of photosensitive materials and UV direct writing techniques will ultimately allow the realisation of high quality integrated Bragg grating and channel waveguiding structures suitable for application within telecomms devices.

\section{(C) IEE 2002}

Electronics Letters Online No: 20021056

25 September 2002

DOI: $10.1049 / \mathrm{el}: 20021056$

G.D. Emmerson, S.P. Watts, C.B.E. Gawith, V. Albanis, M. Ibsen, R.B. Williams and P.G.R. Smith (Optoelectronics Research Centre, University of Southampton, SO17 1BJ, United Kingdom)

E-mail: rbw@orc.soton.ac.uk

\section{References}

1 HLL, K.O., and MELTZ, G.: 'Fiber Bragg grating technology fundamentals and overview', J. Lightwave. Technol., 1997, 15, pp. 1263-1276
2 SVALGAARD, M., and KRISTENSEN, M.: 'Directly UV written silica-onsilicon planar waveguides with low loss', Electron. Lett., 1997, 33, (10), pp. 861-863

3 FAERCH, K., and SVALGAARD, M.: 'Symmetrical waveguide devices fabricated by direct UV writing', IEEE Photonics Technol. Lett., 2002, 14, (2), pp. 173-175

4 UK Patent Application: GB2316760A

\section{Fabrication of low-cost planar wavelength- selective optical add-drop multiplexer by employing UV photosensitivity}

\author{
Mahn Yong Park, Wonshik Yoon, Soowook Han and \\ G. Hugh Song
}

\begin{abstract}
A $2 \times 2$ optical add-drop multiplexer has been fabricated by exclusive use of the UV photosensitivity effect in defining the waveguide patterns and Bragg pattern without using any etching process on the multilayered slab structure prepared by a single session of flame hydrolysis deposition process. That is, the waveguide patterns were written by direct writing of a focused UV beam and, subsequently, the periodic grating was imprinted on the plate by a broad UV exposure over a phasemask. The add and drop efficiencies measured at the Bragg wavelength were 25 and $20 \mathrm{~dB}$, respectively.
\end{abstract}

Introduction: The UV photosensitivity effect in Ge-doped silica fibres has attracted considerable attention since a large refractiveindex increase of the order of $\sim 10^{-3}$ was obtained when the Gedoped silica fibre was illuminated by an intense UV laser beam [1] The effect has also been applied to fabricating various kinds of planar waveguide devices in addition to fibre optic devices. Designed patterns of waveguides have been drawn directly by exposing a focused UV laser beam on the Ge-doped silica plate [2-4]. It was also reported that Bragg grating filters and optical add-drop multiplexers (OADM) were fabricated on the photosensitised silica buriedchannel waveguides that were already formed by the dry etching process of standard photolithography [5].

Here, we made an effort to reduce the production cost of OADMs based on established silica-on-silicon technology in two respects: First, we prepared a three-layered slab waveguide structure in one session of a flame hydrolysis deposition (FHD) process. Secondly, we made exclusive use of the UV photosensitivity effect in defining both the waveguide patterns and Bragg pattern on the multilayered slab structure, eliminating the need for photolithography process associated with dry etching. In this Letter, we report the measured spectrum of the fabricated optical add-drop multiplexer (OADM) evaluating the feasibility of our simplified approach.

Experimental setup: The FHD technique was used in preparing a three-layer silica structure on a silicon substrate for its low-cost massproduction operation. The underclad and overclad layers are borophosphosilicates, in which the dopant concentrations of $\mathrm{B}_{2} \mathrm{O}_{3}$ and $\mathrm{P}_{2} \mathrm{O}_{5}$ were around 20 and $6 \mathrm{~mol} \%$, respectively. In the core layer, about $10 \mathrm{~mol} \%$ of $\mathrm{GeO}_{2}$ was co-doped with about $20 \mathrm{~mol} \%$ of $\mathrm{B}_{2} \mathrm{O}_{3}$. Such relatively high doping concentrations were chosen for the purpose of both enhancing the photosensitivity effect and simplifying the deposition/sintering procedure. The doping concentrations were measured by the electron-probe micro-analyser.

All three silica layers were prepared in one session of the deposition process (continuous deposition) and were sintered simultaneously in an electric furnace. The refractive indices of the clad and core layers of the sample, measured by the prism-coupling method at a wavelength of $1.55 \mu \mathrm{m}$, were 1.4480 and 1.4520 , respectively. The prepared silica plates were then put into a hydrogen-loader of $100 \mathrm{~atm}$ for three days at $50^{\circ} \mathrm{C}$

The UV-writing setup for patterning optical waveguides consists of a frequency-doubled Ar-ion laser operating at the wavelength of $244 \mathrm{~nm}$, several optical components (a beam-expander, an objective lens), and computer-controlled stages with movement accuracy of $50 \mathrm{~nm}$ [2].

By the focused spatially filtered beam from a frequency-doubled Arion laser with optical output power of $80 \mathrm{~mW}$, the designed waveguide patterns were directly UV-written with the scan speed of $30 \mu \mathrm{m} / \mathrm{s}$. From 